Cahiers Société

\title{
Castoriadis et la critique sociologique du sujet transcendantal
}

\section{Thibault Tranchant}

Numéro 1, mai 2019

Castoriadis et les sciences sociales

URI : https://id.erudit.org/iderudit/1068422ar

DOI : https://doi.org/10.7202/1068422ar

Aller au sommaire du numéro

Éditeur(s)

Collectif Société

ISSN

2562-5373 (imprimé)

2562-5381 (numérique)

Découvrir la revue

Citer cet article

Tranchant, T. (2019). Castoriadis et la critique sociologique du sujet

transcendantal. Cahiers Société, (1), 91-123. https://doi.org/10.7202/1068422ar

\section{Résumé de l'article}

La théorie castoriadienne de la connaissance prolonge et renouvelle un des gestes philosophiques de la sociologie, remobilisé ensuite notamment par la phénoménologie, à savoir la réinscription critique du sujet de la connaissance, plus particulièrement le sujet transcendantal, à l'intérieur de ses conditions sociales de possibilité. Dans cet article, la variante castoriadienne de la critique sociologique du sujet transcendantal est examinée. Nous commençons par expliciter les liens qu'établit Castoriadis entre la critique de la " pensée héritée » et celle de la raison sociologique. Ceci étant acquis, nous dégageons les axes centraux de sa critique de la sociologie, base sur laquelle il développe sa propre théorie sociale, la théorie de l’institution imaginaire de la société. Enfin, nous nous tournons vers la spécificité de la critique castoriadienne du sujet transcendantal, qui tient, d'une part, à une problématisation aiguë des rapports entre création et vérité et, d'autre part, à son inflexion politique. 


\title{
Castoriadis et la critique sociologique du sujet transcendantal
}

\author{
Thibault TRANCHANT \\ Université de Rennes 1 et Université de Sherbrooke
}

\section{La critique du sujet transcendantal et la sociologie}

Une voie majeure ${ }^{1}$ de la critique du sujet transcendantal kantien est sa critique sociologique, en particulier celle issue de Marx et de Durkheim. L'objectif principal de la critique sociologique du sujet transcendantal est de restituer ses conditions sociales de possibilité, c'est-à-dire de faire du champ social une condition transcendantale du sujet transcendantal lui-même. Il s'agit, dans ce cas, de définir dans quelle mesure le social détermine les formes et le contenu de la subjectivité transcendantale, impliquant, dès lors, son historicisation et, dans une certaine mesure, sa relativisation. Cette critique sociologique n'est bien entendu pas uniforme, puisqu'elle se diversifie selon les définitions possibles des sciences sociales. Les ontologies du social mobilisées par les auteurs marxistes ou par les auteurs durkheimiens conduisent par exemple à des formulations différentes de l'opération et du contenu de la sociocritique du sujet transcendantal. Quoi qu'il en soit de ces différences internes, c'est au premier tiers du $\mathrm{XX}^{\mathrm{e}}$ siècle qu'on peut dater le plein développement de ce schème critique. L'entre-deux-guerres est en effet marqué par la constitution d'un véritable programme sociologique visant à expliciter les fondements sociaux de la production, de la circulation et de la légitimation de la connaissance - ce qu'on appelle conventionnellement la «sociologie de la connaissance »-, programme de recherche à l'intérieur duquel l'enquête sur les catégories occupe une place centrale (bien que n'étant pas le tout de la sociologie de la connaissance). C'est de cette époque que date Les formes élémentaires de la vie religieuse (1912), texte où Durkheim pose les fondements de l'enquête sociologique sur les origines des catégories mobilisées dans les activités

\footnotetext{
${ }^{1}$ Traversant la modernité postkantienne, une des manifestations majeures de la critique du sujet kantien est le perspectivisme nietzschéen. Une autre est la psychanalyse. Il faut aussi tenir compte, ce qui ne sera pas fait à l'intérieur de cet article, des fines thématisations de la subjectivité à l'intérieur du mouvement phénoménologique, de même qu'il convient de tenir compte de l'enrichissement, par exemple avec Habermas, de la subjectivité à partir de la question du langage et de la communication. Dans une intention qui n'est pas sans faire penser à celle d'Habermas (conserver, contre les formes radicales du postmodernisme philosophique, une dimension transcendantale du sujet tout en l'élargissant au social et à l'histoire), nous pouvons également mentionner les travaux de M. Freitag, notamment dans « La dissolution post-moderne de la référence transcendantale », Cahiers de recherche sociologique, n 33, 1999, p. 181-217.
} 
épistémiques. Mauss, après Durkheim, a approfondi ce programme de recherche, par exemple dans son texte sur la catégorie du moi : «Une catégorie de l'esprit humain : la notion de personne, celle de moi » (1938). C'est aussi à cette époque que paraît $L a$ philosophie des formes symboliques d'Ernst Cassirer (1923-1929), Problèmes de sociologie de la connaissance de Max Scheler (1924) puis Idéologie et utopie de Karl Mannheim (1929). Enfin, c'est de cette époque que date la première conceptualisation rigoureuse d'une critique marxiste du sujet transcendantal, à savoir celle proposée par G. Lukács dans la deuxième partie de son essai sur la réification inclus dans Histoire et conscience de classe (1923). Lukács donne ainsi le coup d'envoi à un véritable programme de sociologie marxiste de la connaissance qui, développé notamment à l'intérieur de l'École de Francfort, trouve en Alfred Sohn-Rethel un de ses théoriciens privilégiés avec son trop méconnu Travail intellectuel et travail manuel : une critique de l'épistémologie (1951, pour la première version). Ainsi, du point de vue du développement interne de l'histoire de la sociologie, la critique de la subjectivité transcendantale occupe une place particulière : non seulement elle doit être située à l'intérieur d'un programme plus large de connaissance sociologique de la connaissance, mais elle prend la forme d'une enquête sur la sociogenèse des catégories mobilisées par le sujet dans l'acquisition ou la production de la connaissance. Ce qu'on peut appeler la « sociocritique du sujet transcendantal » est donc avant tout une sociogenèse de la catégorialité mobilisée par les acteurs de l'activité scientifique².

\section{Castoriadis, la création et la critique sociologique du sujet transcendantal}

La sociocritique du sujet transcendantal est donc largement avancée, sinon acquise, lorsque Castoriadis écrit L'institution imaginaire de la société (1975), livre dans lequel il soutient clairement la thèse d'une détermination sociale des catégories de la

\footnotetext{
2 Il est coutumier de faire de Durkheim, Marx et Weber les trois fondateurs principaux de la sociologie, tout en évoquant leurs prédécesseurs (De Bonald, Saint-Simon, Comte du côté français, par exemple) dans la constitution de leur propre projet scientifique. On s'étonnera dès lors de ne pas voir Weber apparaître dans la «voie sociologique de la critique de la subjectivité transcendantale ». Sans nous engager ici dans un commentaire serré du rapport de Weber au criticisme, l'absence d'une critique explicite de la subjectivité transcendantale chez Weber tient au maintien d'une perspective criticiste forte au niveau de sa conceptualisation de la méthode sociologique ( $C f$. Philippe Raynaud, Max Weber et les dilemmes de la raison moderne, Paris, Presses Universitaires de France, 1987). Cela ne signifie pas que la sociologie wébérienne ne permette pas la critique du sujet kantien, mais seulement qu'on ne trouve pas chez Weber un tel projet, contrairement à Durkheim et, dans une moindre mesure, Marx. De fait, c'est en partie sur certains concepts de la sociologie wébérienne, notamment la typologie idéale des formes de la rationalité, que s'est déployée ensuite dans le "marxisme wébérien » (pour reprendre une appellation de Merleau-Ponty dans Les aventures de la dialectique et reprise par Löwy dans La cage d'acier : Max Weber et le marxisme wébérien) la critique de la subjectivité transcendantale kantienne. Par exemple, la deuxième partie de l'essai sur la réification dans Histoire et conscience de classe de Lukács est presque entièrement dévolue, à travers une critique des antinomies de la pensée bourgeoise, à exposer les rapports réciproques entre réification, capitalisme et criticisme kantien. De même, l'absence, chez Weber, d'une sociogenèse du sujet transcendantal ne signifie pas qu'une sociologie de la connaissance y soit absente.
} 
connaissance. Il y soutient notamment, nous y reviendrons en détail, que les fondements catégoriaux des pratiques épistémiques sont à trouver dans un certain nombre de pratiques sociales, et que les variations historiques de ces fondements sont à rapporter à l'autotransformation de l'«imaginaire social» de chaque unité socialhistorique considérée. Aussi est-il clair pour Castoriadis que les catégories mobilisées par le sujet sont des catégories socialement déterminées. Les catégories de temps ou d'espace, par exemple, ne sont rien, estime-t-il, en dehors de leur inscription à l'intérieur de leur spécification sociale, qui est une spécification imaginaire. Ainsi, sans néanmoins refuser à la subjectivité une certaine indépendance dans la constitution de son expérience, Castoriadis cherche, tout comme le faisaient Durkheim et Marx chacun à leur manière, à réinscrire l'expérience que le sujet fait du monde à l'intérieur de conditions sociales sur lesquelles le sujet n'a pas de contrôle immédiat. Castoriadis réinvestit donc explicitement les traits principaux de la critique sociologique du sujet transcendantal kantien.

Nous sommes donc en droit de nous demander, au regard d'un tel contexte3, quelle est la spécificité de la contribution castoriadienne à ce schème critique. La thèse que nous soutiendrons dans cet article est que la spécificité de sa sociocritique de la subjectivité transcendantale tient à la spécificité de son projet philosophique, à savoir celui d'une critique générale de la raison à partir d'une définition originale de la création comme création ex nihilo de nouvelles déterminations formelles de l'être dans le temps. L'apport de Castoriadis au schème sociocritique de la subjectivité transcendantale ne tient pas à la simple idée que les catégories ont une origine sociale - thèse alors largement acquise -, mais à l'articulation de ce schème critique à un concept radical de création impactant sur la conception qu'il convient de proposer de la subjectivité et de l'objectivité. Pour le dire autrement, l'apport de Castoriadis est, d'un côté, de chercher à penser une certaine modalité de la sociogenèse des catégories d'après son concept de création et, d'un autre côté, d'interroger le rapport d'une telle institution sociocatégorielle à un monde marqué par la contingence et l'imprévisibilité de la création ontologique. Nous verrons que la théorie de l'institution imaginaire de la société est construite par Castoriadis à l'intérieur d'un projet philosophique plus ambitieux, qui consiste à défaire l'emprise de la rationalité logico-mathématique sur celle de la raison en général, notamment afin de pluraliser les formes de la rationalité et de donner à voir des modalités alternatives de la genèse ontologique, en particulier la création ex nihilo. La théorie de l'institution imaginaire de la société de Castoriadis est la dimension sociologique et historique de ce projet philosophique général, et sa sociocritique du sujet transcendantal en est un des aspects internes. Cet article vise donc à expliciter la spécificité de la conception sociogénétique de la catégorialité transcendantale selon Castoriadis, et nous verrons qu'elle tient à son concept de création, qui irrigue l'ensemble de son projet philoso-

\footnotetext{
3 On pourra consulter l'article de Danilo Martuccelli, qui, dans le même numéro, propose une riche
} synthèse de ce contexte théorique. 
phique. Bref, nous examinerons et expliciterons la thèse castoriadienne selon laquelle les formes du sujet transcendantal sont des créations social-historiques, elles-mêmes insérées dans une ontologie de la contingence dont le concept de création est la pièce angulaire.

Nous procéderons en trois temps. Dans un premier temps, nous ferons le point sur les présupposés philosophiques de la critique castoriadienne des sciences sociales. En effet, si Castoriadis remobilise le schème principal de toute sociocritique du sujet transcendantal, à savoir qu'il existe une « construction sociale de la réalité », il reste qu'il le réinscrit dans un projet philosophique plus général en spécifiant le sens. C'est pourquoi nous proposerons, dans un premier temps, une synthèse de la critique castoriadienne de la "pensée héritée », variante originale de la critique postnietzschéenne de la fondation de la philosophie sur les principes de raison suffisante et d'identité. Une fois cette contextualisation philosophique opérée, nous nous tournerons, dans un second temps, vers la critique qu'il propose des sciences sociales à proprement parler. La sociocritique castoriadienne du sujet transcendantal repose en effet sur une critique radicale des sciences sociales et de leur histoire dont le débouché positif est la formulation de la théorie de l'institution imaginaire de la société. Nous examinerons en particulier le schème critique principal de Castoriadis contre les sciences sociales, à savoir qu'elles ne sont guère parvenues, jusqu'à ce jour, à penser, tout d'abord, la spécificité de leur objet d'étude (le social et l'histoire) et, ensuite, la créativité historique comme telle. Bref, nous commenterons sa thèse critique principale à l'encontre des sciences sociales, selon laquelle elles reconduisent en leur sein la "pensée héritée ». Enfin, dans un troisième temps, nous explorerons la pars construens de la critique castoriadienne des sciences sociales dans son rapport à la question de la constitution social-historique de la subjectivité transcendantale. Nous examinerons, en d'autres termes, en quoi la formulation de la théorie de l'institution imaginaire de la société, solution apportée par Castoriadis en vue du renouvellement des sciences sociales au-delà de leur critique, conduit à repenser le schème sociocritique de la subjectivité transcendantale. Nous insisterons sur un aspect original de sa sociocritique du sujet, c'est-à-dire son infléchissement résolument politique.

\section{De la critique de la « pensée héritée » à celle des sciences sociales}

La théorie castoriadienne de l'institution imaginaire de la société, théorie sociologique à l'intérieur de laquelle s'inscrit sa sociocritique du sujet transcendantal, est une facette parmi d'autres d'un projet philosophique plus ambitieux : la critique de la "pensée héritée », définie comme conséquence générale de la fondation, voire de l'identification, de la pensée sur une forme restreinte de rationalité, celle dite « ensembliste-identitaire ». Selon Castoriadis, en d'autres termes, la «pensée héri- 
tée » est celle qui n'interroge pas l'emprunt de ses critères de rationalité à cette forme de rationalité restreinte qu'est la rationalité « ensembliste-identitaire ».

Comprendre l'originalité de la sociocritique castoriadienne du sujet transcendantal requiert donc de saisir, dans un premier temps, la signification de sa critique de la "pensée héritée ». Après avoir fait une synthèse de sa définition de la rationalité ensembliste-identitaire, nous verrons en quoi la «pensée héritée » se définit, selon lui, comme procès d'universalisation de l'ensembliste-identitaire. C'est contre un tel procès, dont la conséquence principale est l'occultation de la création et de sa diversité qualitative, que s'élaborent les tâches de la pensée aujourd'hui et, par conséquent, le renouvellement des sciences sociales.

\section{La rationalité ensembliste-identitaire}

Le concept d' " ensembliste-identitaire » chez Castoriadis est un concept de la logique. Il s'agit d'exhiber les types d'opérations se retrouvant aux fondements de la pensée logique et qu'on peut ensuite identifier dans ses différentes instanciations historiques. Il ne s'agit donc pas de décrire une forme historiquement déterminée de la logique à partir de laquelle se serait bâtie ultérieurement la philosophie, mais plutôt de proposer un inventaire des opérations (des « schèmes » ou " opérateurs fondamentaux ») à partir desquelles se déploient les différentes formes possibles de la logique. C'est la raison pour laquelle nous pouvons dire plus précisément que le concept d'ensembliste-identitaire est un concept praxéologique de la logique, puisqu'il s'agit de qualifier la pensée logique à partir des opérations pratiques qu'elle présuppose. En effet, selon Castoriadis, les schèmes sur lesquels reposent les différentes formes de logique ne sauraient être confondus avec les axiomes d'un système logique ni même être produits par inférence logique à partir des axiomes: leur «application présuppose qu'ils [aient] déjà été appliqué[s4] », ce qui signifie que la logique repose sur une série de "capacités » logiques qu'elle ne fait pas elle-même, mais qui sont nécessaires à son fonctionnement.

Dans L'institution imaginaire de la société, Castoriadis propose une première exploration et catégorisation de ces schèmes pratiques fondamentaux sur lesquels repose la rationalité ensembliste-identitaire. Le premier d'entre eux est celui de la séparation, qui permet d'isoler une portion du réel afin d'en faire un "élément». Il est ce qui permet à la pensée de constituer un objet, de le définir, bref, de lui attribuer une identité. C'est le schème de la séparation qui permet de poser la "pure identité à soi », ainsi que la «pure différence à tout ce qui n'est pas soi 5 ». Ce schème de la séparation est étroitement lié à un autre, celui de la désignation, qui est la capacité fondamentale de désigner une chose comme singulière, comme un ceci. Comment, en effet, séparer sans désigner? Un autre schème pratique sur lequel repose la ratio-

4 Cornelius Castoriadis, L'institution imaginaire de la société, Paris, Seuil, 1999, p. 331.

5 Idem. 
nalité ensembliste-identitaire est celui de la réunion. Il autorise la construction d'ensembles à partir d'entités préalablement identifiées et permet de constituer une « unité identique à soi des différents 6 », c'est-à-dire un ensemble comme objet distinct et défini de type supérieur par rapport aux éléments qui le composent. Dans L'institution imaginaire de la société, Castoriadis nomme aussi, tout en précisant qu'il ne s'agit que d'une "série d'illustrations", les schèmes de la «décomposition», de «l'équivalence opérationnelle propriété $\equiv$ ensemble », de « l'ordre » et de « l'itération7 ». Dans un texte plus tardif, « La logique des magmas et la question de l'autonomie »(1981-1983), Castoriadis propose une description plus synthétique et plus conventionnelle des schèmes pratiques de la rationalité ensembliste-identitaire. Il soutient alors que la rationalité ensembliste-identitaire repose sur les "opérateurs logico-ontologiques fondamentaux» suivants: "principes d'identité, de non-contradiction et du tiers-exclu ; l'équivalence propriété $\equiv$ classe ; l'existence fortement affirmée de relations d'équivalence ; l'existence fortement affirmée de relations de bon ordre ; la déterminité8 $»$.

L'inclusion d'un schème de la "déterminité » dans la définition de la rationalité ensembliste-identitaire peut paraître surprenante, mais lui est pourtant centrale. Dans "Science moderne et interrogation philosophique", Castoriadis définit la « déterminité » comme "immanence à ce qui est de la possibilité d'être défini et distingué9 », c'est-à-dire présupposition de l'adéquation entre l'être en sa totalité et les opérateurs fondamentaux de la rationalité ensembliste-identitaire. En d'autres termes, la rationalité ensembliste-identitaire n'est pas simplement une logique, mais est aussi, inséparablement, une ontologie : la détermination ensembliste-identitaire de la rationalité est aussi phénoménalisation ensembliste-identitaire du réel ; la position du concept (ensembliste-identitaire) est celle de l'être (ensembliste-identitaire). C'est la raison pour laquelle la

logique identitaire [...] vaut décision ontologique sur ce qui est et la façon dont il est : ce qui est est tel qu'il y existe des ensembles (des choses et des relations identitaires). Décision qui est en même temps expression d'une création, d'une genèse ontologique : des ensembles, ces ensembles et l'eidos d'ensemble sont désormais posés-institués et comme tels sont dans une nouvelle région d'être10.

\footnotetext{
6 Ibid., p. 332.

7 Ibid., p. 333.

8 Cornelius Castoriadis, « La logique des magmas et la question de l'autonomie », dans Les carrefours du labyrinthe, II. Domaines de l'homme, Paris, Seuil, 1999, p. 487.

9 Cornelius Castoriadis, "Science moderne et interrogation philosophique », dans Les carrefours $d u$ labyrinthe, I, Paris, Seuil, 1986, p. 268.

10 Cornelius Castoriadis, L'institution imaginaire de la société, op. cit., p. 335.
} 
Pour reprendre une expression deleuzienne, nous pourrions dire que la rationalité ensembliste-identitaire est une « image de la pensée », à savoir une détermination transcendantale et universelle de l'être à partir d'une création contingente de la pensée humaine.

Enfin, la rationalité ensembliste-identitaire se distingue par la modalité spécifique du sens qu'elle véhicule : la signification ensembliste-identitaire est combinatoire. Ne font sens à l'intérieur de la rationalité ensembliste-identitaire que les propositions construites à partir des opérateurs logiques fondamentaux que nous venons de décrire :

Thèse : la signification au sens ensembliste-identitaire est réductible à des combinaisons de ces deux acceptions du «sens" [ici, Castoriadis désigne le sens comme inclusion dans une classe et le sens comme mise en relation] - et réciproquement : toute signification réductible à des combinaisons de ces deux acceptions du « sens » est ensembliste-identitaire. Autrement dit: les énoncés significatifs au sens ensembliste-identitaire concernent toujours les inclusions dans des classes, les insertions dans des relations, et la combinatoire qui peut être construite là-dessus.

Autre formulation de la thèse: les significations au sens ensembliste-identitaire sont constructibles par classes, propriétés et relations («par figures et mouvements », aurait dit Descartes).

Corollaire de la thèse : il existe des significations qui ne sont pas constructibles par classes, propriétés et relations ${ }^{11}$.

\section{La pensée héritée comme universalisation de la rationalité ensembliste- identitaire et ses conséquences}

Si la rationalité ensembliste-identitaire est une forme particulière de rationalité, l'histoire de la rationalité occidentale se caractérise selon Castoriadis par l'identification de la raison à la rationalité ensembliste-identitaire. C'est en ce sens qu'il est permis de dire qu'il propose une variante de la critique postnietzschéenne de la philosophie, puisqu'il s'agit d'ouvrir la philosophie à de nouveaux possibles en la rapportant à une série de décisions épistémiques contingentes. Quoi qu'il en soit, Castoriadis ne critique pas la rationalité ensembliste-identitaire comme telle. Il critique les conséquences, au niveau de la pensée et de la pratique, d'un développement historique ayant conduit à la fondation unilatérale de la raison en général sur la rationalité ensembliste-identitaire :

${ }^{11}$ Cornelius Castoriadis, «La logique des magmas et la question de l'autonomie », dans Les carrefours du labyrinthe, II, op. cit., p. 503. 
À cette nécessité, transhistorique [celle de création de l'ensembliste-identitaire par chaque société], s'ajoute, pour nous, un développement historique particulier, et que l'on peut penser dépassable: le tour spécifique qu'a pris la philosophie depuis Parménide et surtout Platon comme ontologie de la déterminité, soit comme dilatation exorbitante de l'ensembliste-identitaire, recouvrant presque tout le domaine de la pensée, constituant aussi une «philosophie politique rationnelle », pour finalement aboutir - moyennant certes aussi d'autres apports - au règne de la pseudo-« rationalité » que nous connaissons dans le monde moderne ${ }^{12}$.

Aussi la fondation de la raison sur la rationalité ensembliste-identitaire est-elle universalisation de sa modalité spécifique de signification et de sa conception de l'être. C'est cette universalisation que Castoriadis désigne comme "pensée héritée », et ce sont ses conséquences qu'il critique.

La plus importante de ces conséquences est l'occultation du « fait de la création », c'est-à-dire l'invisibilisation d'une modalité spécifique de la genèse ontologique: l'émergence acausale de l'altérité formelle dans le temps. La rationalité ensemblisteidentitaire sur laquelle se fonde la pensée héritée ne dispose d'aucune ressource afin de penser ce fait ontologique massif que serait l'émergence de nouvelles formes de l'être à travers le temps. Pour Castoriadis, la «création » n'est pas à comprendre selon sa définition d'origine grecque comme poièsis. Elle n'est pas l'imposition d'une forme donnée préalablement à une matière qui s'y prête (à la manière du démiurge dans le Timée). La «création», au sens strict, est, selon sa définition d'origine judaïque (Castoriadis ne revendique pas explicitement cette origine conceptuelle), l'émergence « ex nihilo» d'une nouvelle forme de l'être dans le temps, émergence d'un nouvel eidos, c'est-à-dire d'un nouveau principe unitaire de détermination d'une multiplicité qui n'était aucunement donné dans ce qu'il organise. «La création veut dire précisément la position de nouvelles déterminations - l'émergence de nouvelles formes, eidé, donc ipso facto l'émergence de nouvelles lois - les lois qui appartiennent à ces modes d'être13. » Aussi la genèse de cette " nouvelle détermination » n'est-elle pas un effet de la multiplicité qu'elle détermine : la forme comme principe de détermination n'est pas déterminée par ce qu'elle détermine. "Comme tel, le nouvel eidos, la nouvelle forme, est créé ex nihilo. En tant que forme, en tant qu'eidos, il n'est pas productible ou déductible de ce qui "était làı" ». Sur le plan conceptuel, Castoriadis fait une distinction entre " altérité » et " différence » afin de

12 Ibid., p. 508. Nous soulignons.

13 Cornelius Castoriadis, "Fait et à faire», dans Les carrefours du labyrinthe, V. Fait et à faire, Paris, Seuil, 2008, p. 20.

14 Cornelius Castoriadis, «Temps et création », dans Les carrefours du labyrinthe, III. Le monde morcelé, Paris, Seuil, 1990, p. 334. 
rendre compte de la spécificité de la création comme modalité de la genèse ontologique. « Deux objets sont différents s'il existe un ensemble de transformations déterminées ("lois") permettant la déduction ou production de l'un à partir de l'autre. Si un tel ensemble de transformations déterminées n'existe pas, les objets sont autres'15. » L'existence de l'altérité ontologique, qui est une altérité formelle, est pour Castoriadis un fait incontestable : nous ne pouvons pas ne pas penser l'expérience du réel en dehors de la catégorie de l'altérité. Or, d'après lui, la rationalité ensemblisteidentitaire n'est d'aucun secours afin de penser la genèse de l'altérité formelle : elle ne permet de penser que la différence. En effet, les schèmes logiques de la succession dont dispose la pensée héritée, c'est-à-dire les schèmes logiques tels que la causalité ou l'implication logique à partir desquels est conceptualisée la genèse ontologique, ne permettent de penser que le développement d'une identité à travers le temps. Au même titre que l'implication logique, la causalité est une identité développée : l'effet est contenu dans la cause et, comme tel, ne débouche aucunement sur une nouvelle configuration ontologique, mais seulement sur une configuration ontologique différente. D'un point de vue strictement ontologique, rien, en effet, n'est ajouté dans le monde à la suite d'une relation causale. La relation causale n'est pas une création ; elle est, selon Castoriadis, une différenciation, c'est-à-dire une reconfiguration d'un état donné de l'être dans le temps. Il en va de même pour les autres schèmes logiques de la succession dont dispose la pensée héritée.

Castoriadis ne veut pas dire que la rationalité ensembliste-identitaire n'explique rien. Au contraire, son efficience n'est plus à démontrer, du fait, selon lui, de la forte adéquation de l'ensembliste-identitaire à une part importante du réel " en soi ». La rationalité ensembliste-identitaire "s'étaye " (le concept est repris par Castoriadis à la métapsychologie freudienne, qui permet de désigner la relation des représentations psychiques au biologique) à un réel en partie conforme aux critères de vérité de la rationalité ensembliste-identitaire. Mais, une fois ceci établi, Castoriadis fait remarquer que le réel, comprenant la question de la genèse ontologique, ne se réduit pas à ce que la rationalité ensembliste-identitaire peut en dire, et c'est le tort de la "pensée héritée » que de l'affirmer. La création, comme modalité de la genèse, fait partie de cette « part » du réel que la rationalité ensembliste-identitaire ne parvient pas à exprimer, mais qui appartient pourtant à l'être « en soi », c'est-à-dire à l'être tel qu'il est indépendamment de ce que nous en pouvons dire dans l'état actuel de notre savoir.

\section{Les tâches de la pensée aujourd'hui : raison et création}

L'occultation de la création par la pensée héritée appelle donc une reconstruction de la pensée et de la pratique : «il faut casser l'idée de la déterminité, c'est-à-dire de

15 Ibid., p. 333. 
l'être comme être déterminé, et revoir que l'être est création ${ }^{16}$ ». La reconstruction de la pensée autour de la création se développe chez Castoriadis selon plusieurs axes :

1. La question ontologique : comment penser l'être « en soi » comme créateur?

2. La question épistémologique : comment penser les conditions de la connaissance de l'être par le sujet une fois considéré qu'il est le créateur de ses propres déterminations de connaissance?

3. La question de leur co-implication : comment penser la relation entre création ontologique et auto-institution de la catégorialité par le sujet? C'est la question de l'histoire de la science et de la vérité.

4. La question politique : comment agir dans un monde créateur?

Les aspects les plus connus de l'œuvre de Castoriadis (théorie de la démocratie, conceptualisation de l'autonomie, théorie de l'imaginaire social et de l'imagination radicale, théorie de la psyché, concept ontologique de «magma », etc.) sont des propositions conceptuelles afin de répondre positivement à la problématique plus générale de la reconstruction de la raison à partir de la question de la création ${ }^{17}$. La théorie de l'institution imaginaire de la société est quant à elle l'aspect sociologique et historique de ce projet plus global de reconstruction de la raison à partir de la notion de création, et elle est, à ce titre, concernée par les quatre axes que nous venons d'identifier, puisqu'elle mobilise aussi bien une problématique ontologique qu'épistémologique (ainsi que leurs rapports) et politique. Aussi la reconstruction des sciences sociales et des sciences historiques à partir de la notion de création, qui est réélaboration de la forme de rationalité devant leur correspondre, a-t-elle pour corrélat, sinon pour condition, la critique des savoirs déjà constitués sur le social et l'histoire. C'est ce que nous pouvons désormais examiner, après quoi nous verrons quelles en sont les conséquences pour la sociocritique du sujet transcendantal.

\section{Critique de la « pensée héritée » dans les sciences sociales}

Castoriadis remobilise les traits principaux de la sociocritique du sujet transcendantal, notamment dans sa variante «holiste». D'un côté, il commence par affirmer qu'on ne saurait penser le social que comme domaine de l'être sui generis auquel

\footnotetext{
16 Cornelius Castoriadis, Sur « Le politique » de Platon, Paris, Seuil, 1999, p. 106.

17 Notons cependant que du point de vue de la genèse de l'œuvre, l'exigence d'une reconstruction générale de la raison à partir de la notion de création est acquise au terme d'une réflexion plus circonstanciée sur la spécificité ontologique du social-historique et sur les méthodes susceptibles d'en rendre compte, notamment le matérialisme historique dans sa variante marxiste-léniniste. Suzi Adams a très bien souligné cette progression interne de l'œuvre de Castoriadis (allant de la question social-historique à une réflexion d'ordre cosmologique) dans son remarquable Castoriadis's ontology : being and creation, New York, Fordham University Press, 2011.
} 
doit correspondre une méthodologie spécifique ${ }^{18}$. D'un autre côté, il soutient que le point de vue du social-historique nouvellement acquis est celui à partir duquel il faut reprendre les problèmes de la philosophie, notamment ceux en rapport à la constitution du sujet et du savoir scientifique.

Toutefois, cette identité structurelle ne signifie pas qu'il se contente de répéter, pour parler comme Nisbet, la « tradition sociologique ». Il entend, au contraire, construire sa propre théorie sociologique, la théorie de l'institution imaginaire de la société, contre la sociologie. Castoriadis considère en effet que l'occultation par toutes les sociologies du «fait de la création » ruine leur prétention à la scientificité. Ainsi, la critique des sciences sociales par Castoriadis n'est pas une simple répétition du geste critique ayant rendu possible la sociologie comme discipline autonome, mais reprise de ses problématiques à partir d'une refondation de l'ontologie sur la notion de création. Ce qui est en jeu dans une telle reprise est à la fois le statut scientifique des sciences sociales et, pour ce qui nous concerne plus particulièrement ici, la question de l'autoconstitution du sujet transcendantal à partir d'une conception renouvelée de la nature et de l'objet des sciences sociales.

Conformément à son projet philosophique, il s'agit donc pour Castoriadis de proposer une critique de la pensée héritée dans les sciences sociales. D'une part, il s'interroge sur les conséquences de la fondation des sciences sociales et historiques sur les opérateurs logico-ontologiques distinctifs de la rationalité ensemblisteidentitaire (ce qui en fait une ramification de la "pensée héritée »). D'autre part, il s'agit de repenser les sciences sociales et historiques à partir de ce qu'elles auraient exclu jusqu'à maintenant, à savoir la création et la modalité ontologique spécifique de l'être social-historique ${ }^{19}$. Dans ce qui suit, nous apprécierons la critique castoriadienne des sciences sociales en reconstituant, dans un premier temps, les tâches qu'il assigne à la pensée sociale et historique, pour, ensuite, examiner les impacts de la fondation implicite des sciences sociales sur la « pensée héritée ».

\footnotetext{
18 Voir, dans ce numéro, l'article de Geneviève Gendreau.

19 La littérature secondaire sur la critique castoriadienne des sciences sociales n'est pas très abondante. On pourra lire : Gérald Berthoud, "Castoriadis et la critique des sciences sociales », dans Autonomie et autotransformation de la société : la philosophie militante de Cornelius Castoriadis, dir. Giovanni Busino, Genève, Droz, 1989, p. 441-456 ; Danilo Martuccelli, "Cornelius Castoriadis : promesses et problèmes de la création ", Cahiers internationaux de sociologie, vol. 2, n ${ }^{\circ} 113,2002$, p. 285-305; Mathieu Noury, « Cornélius Castoriadis, sociologue ? Critique sociologique de l'ontologie de la création imaginaire sociale », Aspects sociologiques, vol. 18, $\mathrm{n}^{\mathrm{o}} 1,2011$, p. 1-19; Hans Joas, «L'institutionnalisation comme processus créateur. Sur la signification sociologique de la philosophie politique de Cornelius Castoriadis », dans Autonomie et autotransformation de la société : la philosophie militante de Cornelius Castoriadis, op. cit., p. 173-190; Axel Honneth, « Une sauvegarde ontologique de la révolution. Sur la théorie sociale de Cornelius Castoriadis ", dans Autonomie et autotransformation de la société, op. cit., p. 191-208 ; Javier Cristiano, Lo social como institución imaginaria. Castoriadis y la teoría sociológica, Villa Maria, Eduvim, 2009.
} 


\section{Les tâches des sciences sociales et leur histoire}

La critique des sciences sociales et historiques par Castoriadis repose sur une série de postulats sur les tâches qu'elles sont censées accomplir. D'après lui, en effet, toute pensée du social et de l'histoire doit répondre à ces trois questions principales :

1. La question de la définition du mode d'être du social.

2. La question de l'unité d'une formation sociale historiquement déterminée.

3. L'élucidation de l'histoire comme création de nouvelles formes d'organisations sociales.

Selon Castoriadis, il s'agit là de son expression, il y a deux « noyaux » de la réflexion sociohistorique :

Qu'est-ce que la société ; notamment, qu'est-ce que l'unité et l'identité (eccéité) d'une société, ou, qu'est-ce qui tient une société ensemble?

Qu'est-ce que l'histoire ; notamment, comment et pourquoi y a-til altération temporelle d'une société, en quoi est-elle altération, y a-t-il émergence du nouveau dans cette histoire, et que signifie-telle 20 ?

En d'autres termes, la tâche de toute réflexion sur le social devrait être, sur la base d'une appréciation préalable de la nature ontologique du social, de saisir ce qui fait l'unité spécifique d'une formation sociale, de même que celle de toute réflexion sur l'histoire devrait être de penser les raisons de l'altération d'une formation sociale en une autre («formation sociale » au sens fort d'eidos social-historique, c'est-à-dire de principe ontologiquement et logiquement différencié de totalisation des interactions et institutions individuelles ${ }^{21}$ ). L'histoire des sciences sociales serait celle des réponses complémentaires ou contradictoires apportées à ces questions.

C'est donc d'après ce postulat que Castoriadis développe sa critique des sciences sociales. Elle consiste principalement à désigner l'écart qui existerait entre, d'un côté, les tâches qu'elles sont censées accomplir et, d'un autre côté, l'incapacité des savoirs constitués afin de les résoudre. Dès lors, sa thèse, on le devine, est que l'origine d'un tel écart est l'occultation de la création par les principes logiques et ontologiques de la «pensée héritée ». Naturellement, la contrepartie de cette thèse critique est celle, contraire, selon laquelle on ne peut satisfaire les fins de la réflexion sociale et historique qu'à condition de la recentrer sur la notion de création.

\footnotetext{
20 Cornelius Castoriadis, L'institution imaginaire de la société, op. cit., p. 255.

${ }^{21}$ Cf. Sophie Klimis, "Créer un eidos du social-historique selon Castoriadis », dans Affectivité, imaginaire, création sociale, Bruxelles, Facultés universitaires Saint-Louis, 2010, p. 13-42.
} 
En ce qui concerne l'histoire des sciences sociales, Castoriadis considère qu'il n'existe pas de savoirs sur le social et l'histoire qui ne se soient pas fondés ou qui n'aient pas cherché à se dégager des présupposés logico-ontologiques de la rationalité ensembliste-identitaire ${ }^{22}$. Il classe l'ensemble des savoirs sociohistoriques en deux catégories distinctes. La première est la catégorie "physicaliste», dans laquelle le social et l'histoire sont pensés d'après le modèle des sciences de la nature, réinvestissant ainsi la forme particulière de rationalité leur correspondant - essentiellement causaliste et régie par le principe de raison suffisante. La forme la plus pure en serait le fonctionnalisme, qui se donne une série de besoins biologiques et transhistoriques auxquels les institutions sociales sont censées donner satisfaction ${ }^{23}$. Ainsi, dans le cas $\mathrm{du}$ fonctionnalisme, " on recouvre [...] le fait essentiel : les besoins humains, en tant que sociaux et non simplement biologiques, sont inséparables de leurs objets, et les uns comme les autres sont chaque fois institués par la société considérée 24 ». Le physicalisme sociohistorique, qui est un naturalisme et dont le fonctionnalisme est une variante, occulte ainsi la création social-historique. La seconde est la catégorie « logiciste ». Dans cette catégorie, les phénomènes sociaux et historiques sont expliqués directement à partir des catégories abstraites de la pensée logique. Cette fois, les types les plus purs en seraient la rationalité dialectique d'origine hégélienne et le structuralisme d'origine lévi-straussienne 25 . Qu'il s'agisse du "physicalisme » ou du «logicisme», Castoriadis soutient qu'aucune de ces approches du social et de l'histoire ne permet de résoudre les tâches constitutives des sciences sociales et historiques. La question de la spécificité de l'être social n'y est pas résolue, puisque le social est pensé à partir de ce qu'il n'est pas (la fonction, la dialectique, la structure, etc.). La question de l'histoire n'y est pas non plus abordée, puisque le temps y est pensé à partir des schèmes de la succession logique, qui sont des schèmes de l'identité et de la différence, non de l'altérité.

Ainsi, la question de l'unité et de l'identité de la société et de telle société est ramenée à l'affirmation d'une unité et identité données d'un ensemble d'organismes vivants; ou d'un hyper-organisme comportant ses propres besoins et fonctions; ou d'un groupe naturel-logique d'éléments; ou d'un système de déterminations rationnelles. De la société comme telle il ne reste, dans tout cela, rien; rien qui soit l'être propre du social, qui manifeste un mode d'être différent de ce que nous savions déjà par ailleurs. Il ne reste pas non plus grand-chose de l'histoire, de l'altération temporelle produite dans et par la société. Devant la question de l'histoire, le physicalisme devient naturellement causalisme, à savoir suppression de la question. Car la question de l'histoire est question

\footnotetext{
22 Cornelius Castoriadis, L'institution imaginaire de la société, op. cit., p. 254-264.

23 Ibid., p. 255-256.

24 Ibid., p. 256.

25 Ibid., p. 256-258.
} 
de l'émergence de l'altérité radicale ou du nouveau absolu [...]; et la causalité est toujours négation de l'altérité, position d'une double identité; identité dans la répétition des mêmes causes produisant les mêmes effets, identité ultime de la cause et de l'effet puisque chacun appartient nécessairement à l'autre ou les deux à un même 26 .

Ce qui fait donc défaut à l'ensemble des sciences sociales et historiques est la problématisation d'un écart possible entre l'être du social-historique et les catégories abstraites de la pensée censées l'exprimer. Pour ces formes de la pensée héritée dans les sciences sociales et historiques, le social et l'histoire sont approchés d'après le présupposé distinctif selon lequel il y a immanence des déterminations de la rationalité ensembliste-identitaire à l'être dans sa totalité.

\section{L'occultation du social-historique dans les sciences sociales et historiques : le cas de l'Un social-historique}

Cette critique peu généreuse de l'histoire des sciences sociales ${ }^{27}$ se comprend mieux si on considère plus en détail un des aspects centraux de la critique par Castoriadis des sciences sociales, à savoir celle des conceptualisations sociologiques de l'unité et de la totalité social-historiques.

\section{Ensembliste-identitaire et unité social-historique}

Selon Castoriadis, un des aspects distinctifs de la pensée héritée est l'universalisation qu'on y trouve des schèmes fondamentaux de la rationalité ensembliste-identitaire, c'est-à-dire que ses schèmes fondamentaux et concepts logiques sont censés valoir à tous les niveaux de l'être. Un des cas particulièrement révélateurs de cette universalisation s'observe dans les différentes réponses ayant été apportées à la question de l'unité social-historique, qui est celle consistant à déterminer ce qui fait qu'une société tient non seulement comme un tout, mais aussi comme ce tout particulier. Si on examine l'histoire de la théorie sociologique et historique, on y constate en effet que la question de l'unité social-historique y a toujours été pensée à partir d'une conception logique ou mathématique de l'unité : les «schèmes de la coexistence » sont empruntés à ceux de la rationalité ensembliste-identitaire. Or, nous ne saurions penser l'unité social-historique, selon Castoriadis, comme unité numérique, c'est-à-dire comme unité indivisible, ou comme unité spécifique, c'est-à-dire comme unité divisible d'éléments partageant des propriétés communes. Au contraire, le socialhistorique, en tant que domaine sui generis de l'être, requiert non seulement un

26 Ibid., p. 258-259.

27 On pourra lire, avec une bonne présentation de la critique castoriadienne du matérialisme historique : Philippe Caumières, "Le social-historique chez Castoriadis et le poids de la pensée héritée », dans Cahiers Castoriadis, II. Imaginaire et création historique, dir. Philippe Caumières, Sophie Klimis, Laurent Van Eynde, Bruxelles, Facultés Universitaires Saint-Louis, 2006, p. 31-64. 
concept général d'unité non numérique lui étant propre, mais chaque société particulière dans l'histoire requiert à son tour la formulation d'un concept d'unité spécifique. D'où une double exigence épistémologique pour la résolution de la question de la totalité social-historique d'après Castoriadis : produire une conceptualité adéquate à l'étude du mode d'être spécifique de l'unité social-historique et la raffiner en fonction de chaque société considérée (chaque société instituant sa forme particulière de coexistence des éléments la constituant).

La société se donne immédiatement comme coexistence d'une foule de termes ou d'entités de différents ordres. De quoi dispose donc la pensée héritée pour penser une coexistence, et le mode d'être-ensemble d'une diversité de termes ?

Ou bien cette coexistence, cet être-ensemble d'une diversité, est considéré comme un système réel, quelle que soit sa complexité. Il doit y avoir alors possibilité de décomposition effective (réelle ou idéale-abstraite) du système en sous-systèmes bien définissables, en parties et finalement en éléments provisoirement ou définitivement derniers. Ces éléments, bien distincts et bien définis, doivent être susceptibles de définition univoque. Ils doivent être reliés entre eux par des relations de détermination causale, linéaire ou cyclique (réciproque), catégorique ou probabiliste - relations elles aussi susceptibles de définition univoque ; et des relations du même type doivent valoir entre parties, sous-systèmes, etc., du système global. Il en résulte aussi qu'il doit y avoir possibilité de recomposition (réelle ou idéale-abstraite) sans excès ni défaut du système à partir de ses éléments et de ces relations, considérés comme seuls à posséder une réalité dernière. - $\mathrm{Ou}$ bien, l'être-ensemble de la diversité est celui d'un système logique (au sens large, incluant la mathématique). Dans ce cas encore, il doit y avoir position d'éléments derniers, bien distincts et bien définis, définis de manière univoque, et de relations univoques entre ces éléments.

Dans les deux cas, ce qui est à l'œuvre est la logique ensemblisteidentitaire. Dans les deux cas, la société est pensée comme ensemble d'éléments distincts et définis, se rapportant les uns aux autres par des relations bien déterminées. Dans la mesure où [...] la société est tout autre chose qu'un ensemble ou une hiérarchie d'ensembles, il est exclu qu'on puisse en penser, sur cette voie, quelque chose d'essentiel28.

28 Cornelius Castoriadis, L'institution imaginaire de la société, op. cit., p. 264-265. 
Ainsi, l'ontologie du social-historique proposée par la pensée héritée est une ontologie dérivée de celle véhiculée par la rationalité ensembliste-identitaire (dans le sens où l'être y est universellement conçu à partir de ses critères de rationalité), laquelle n'est pas compatible avec une conception pluraliste de l'ontologie pour laquelle il existe non seulement des régions de l'être irréductibles les unes aux autres, mais aussi des formes de légalité et d'organisation auxquelles doit correspondre la création de systèmes catégoriaux inédits et irréductibles aux schèmes de la rationalité ensembliste-identitaire. La pensée héritée est un réductionnisme logique universel qui ne se sait pas toujours comme tel et, quand bien même nous trouverions dans les sciences sociales et historiques l'affirmation ontologique de l'existence de la région « social», l'étude de celle-ci se fait à travers les schèmes opérateurs dérivés de la rationalité ensembliste-identitaire.

\section{Sur la distinction entre holisme et individualisme}

Cette thèse interprétative de Castoriadis s'applique assez aisément au cas de l'individualisme méthodologique, et on pourrait croire pour cette raison qu'elle ne concerne pas la variante «holiste » de l'histoire des sciences sociales et historiques. Pourtant, Castoriadis prend bien soin de souligner que sa critique des sciences sociales et historiques concerne aussi les différentes formes d'holisme sociologique. Il soutient en effet que les sociologies holistes ont été jusqu'à ce jour des réductionnismes inconscients : "Mais lorsque l'on y regarde de plus près, l'on constate non seulement que rien n'est dit [dans les conceptions holistes du social et de l'histoire] sur ce qui resterait ainsi irréductible, mais qu'en fait cet irréductible est en réalité réduit29. » Ainsi, le social serait rapporté à l'individu comme cause finale ou efficiente chez Aristote, aux rapports et moyens de production dans la pensée marxienne ou à la psyché individuelle chez Freud ${ }^{3}$. Il en irait de même dans le cas des pensées sociologiques faisant intervenir des concepts tels que l'«inconscient collectif» ou la " conscience collective », " métaphores illégitimes [...] dont le seul signifié possible est le problème même ici discuté31 ». Si une des tâches de la raison sociologique est de penser la catégorie générale de l'unité sociohistorique à partir du social-historique lui-même, alors le débat entre individualisme et holisme devient un faux débat, puisqu'il n'apparaît que comme une fausse transposition de la définition logicomathématique de l'unité dans le champ social-historique. Qu'on pense le social comme effet de l'interaction de la multiplicité qui le compose ou bien comme principe de totalisation apriorique à partir duquel doit être pensée l'interaction sociale, le point de vue sur le social reste le même : un point de vue "extérieur » fondé sur les schèmes de la rationalité ensembliste-identitaire. La tâche de la pensée socialhistorique n'est pas de conceptualiser dans l'abstrait le rapport des individus ou des

\footnotetext{
29 Ibid., p. 265.

30 Ibid., p. 265-266.

31 Ibid., p. 267.
} 
institutions à la totalité, mais le type chaque fois spécifique de coexistence de la multiplicité dans une unité social-historique chaque fois singulière. De ce point de vue, individualisme et holisme échouent tous les deux, puisqu'ils pensent la coexistence de la multiplicité à partir de schèmes logiques n'appartenant pas au social-historique lui-même.

La critique de Castoriadis à l'égard des sociologies holistes paraît outrancière. Durkheim, par exemple, pose au principe de sa démarche scientifique la thèse de l'existence du social comme région spécifique de l'être, existence à laquelle doit correspondre la formulation d'une méthode distincte afin d'en restituer la légalité32. Mais nous comprenons mieux la critique de Castoriadis lorsque nous nous penchons plus précisément sur les formes de rationalité investies par Durkheim afin d'expliquer le social. Chez celui-ci, en effet, la thèse d'une existence sui generis du social n'est pas accompagnée d'une interrogation en profondeur sur les rapports entre le social comme modalité de l'être et les conventions logiques dont nous disposons afin de l'exprimer. Par exemple, l'explication sociologique chez Durkheim consiste principalement à recomposer des rapports causaux entre des éléments construits préalablement (la "constitution des types sociaux») sur le modèle des sciences de la nature. Selon Castoriadis en d'autres termes, l'affirmation d'une existence du social comme mode de l'être, s'il s'agit assurément d'une avancée scientifique, cache toujours la misère d'un usage non problématisé de la rationalité ensembliste-identitaire. Aussitôt l'être du social affirmé, les sociologues, même " holistes", se seraient empressés d'en recouvrir la spécificité ontologique par la permanence, dans leurs constructions méthodologiques, des schèmes et fondements de la pensée héritée.

Dès lors, qu'elle l'ait su ou non, voulu ou non, et même dans les cas où elle a pu viser explicitement le contraire, la pensée héritée a été nécessairement amenée à réduire le social-historique aux types primitifs d'être qu'elle connaissait ou croyait connaître [...] par ailleurs, à en faire une variante, une combinaison ou une synthèse des étants correspondants : chose, sujet, idée ou concept. Dès lors aussi, société et histoire se trouvaient subordonnées aux opérations et fonctions logiques déjà assurées, et paraissaient pensables au moyen des catégories établies en fait pour saisir quelques existants particuliers, mais posées par la philosophie comme universelles $[\ldots]$.

Mais si l'on décide de considérer le social-historique pour luimême ; si l'on comprend qu'il est à interroger et à réfléchir à partir de lui-même; si l'on refuse d'éliminer les questions qu'il pose en le soumettant d'avance aux déterminations de ce que nous connaissons ou croyons connaître par ailleurs - alors on constate

${ }^{2} C f$. Émile Durkheim, Les règles de la méthode sociologique, Paris, Presses Universitaires de France, 2007 [1895]. 
qu'il fait éclater la logique et l'ontologie héritées. Car on s'aperçoit qu'il ne tombe pas sous les catégories traditionnelles, sauf nominalement et à vide, qu'il force plutôt à reconnaître les limites étroites de leur validité, qu'il permet d'entrevoir une logique autre et nouvelle et, par-dessus tout, force à altérer radicalement le sens de : être33.

Nous comprenons mieux, au regard de cette critique, pourquoi Castoriadis a également été aussi hostile à l'encontre des différentes formes de rationalité dialectique issues de la dialectique hégélienne34. En effet, la dialectique est une forme de panlogisme ensembliste-identitaire, dans le sens où elle n'interroge pas l'écart toujours possible entre les formes de légalité surgissant de l'autopoïèse ontologique et la création par le sujet de sa propre catégorialité. Pour Castoriadis, la dialectique dans sa variante hégélienne, puis marxienne, exhibe les caractères de la pensée héritée au plus haut point, puisqu'il s'agit de penser l'être universellement à partir d'un ensemble de déterminations logiques sans même questionner le présupposé selon lequel les lois dialectiques puissent être celles de l'ontogenèse. À travers la dialectique, il ne reste, dès lors, plus rien du social-historique, celui-ci étant pensé à partir de ce qu'il n'est pas en lui-même, à savoir un ensemble de médiations logiques censé exprimer les lois de son ontogenèse, notamment la négation et la contradiction. Cela ne signifie pas, par ailleurs, qu'il faille abandonner la question du « concret», à savoir celle de la formulation d'une forme de rationalité permettant d'exprimer la totalité ontologique, mais plutôt qu'elle doit être reprise à partir du point de vue de la diversité régionale de l'être, qui est, pour le sujet, diversité des formes de rationalité : la totalisation épistémologique d'un «monde morcelé » ne peut se faire à partir d'une forme de rationalité, la dialectique, dont un des effets est l'écrasement des différentes formes de donation de la signification.

La création ontologique est celle de formes de l'être, et la création de formes de l'être est chaque fois création d'une légalité spécifique. Le "social-historique » est précisément une de ces formes de l'être émergeant au niveau cosmologique et, à ce titre, il possède une légalité intrinsèque et irréductible aux autres formes de l'être. La tâche du sujet de la connaissance, en tant qu'il lui revient celle de créer ses propres déterminations de pensée, est d'adapter son activité rationnelle à l'objet qu'il étudie. Dans le cas de l'étude du social-historique, il lui faut par conséquent créer une forme de rationalité qui corresponde au plus près à la spécificité ontologique de l'êtresocial. Selon Castoriadis, cet effort de création conceptuelle et méthodologique n'a pas encore été adéquatement fourni, malgré les tentatives diverses et variées ayant été faites à cette fin.

\footnotetext{
33 Cornelius Castoriadis, L'institution imaginaire de la société, op. cit., p. 253-254.

34 Il s'agit là, au-delà de certaines convergences, d'une des lignes de démarcation les plus importantes entre Castoriadis et Freitag.
} 
La théorie de l'institution imaginaire de la société est la proposition conceptuelle de Castoriadis à ce problème ontologique et épistémologique, et elle est le cadre dans lequel se développe sa sociocritique du sujet transcendantal.

\section{Théorie de l'institution imaginaire de la société et sociogenèse du sujet transcendantal}

La sociocritique du sujet transcendantal par Castoriadis présente les traits généraux de celle héritée de la tradition sociologique : les catégories de la connaissance mobilisées par le sujet sont celles mises à disposition par la société. Cependant, elle s'en distingue en raison de son ancrage sur son ontologie de la contingence et du socialhistorique, elle-même centrée autour de la notion d'imaginaire. Nous allons désormais nous intéresser à la pars construens de la sociocritique du sujet transcendantal par Castoriadis. Dans un premier temps, nous allons commenter les rapports entre la théorie de l'institution imaginaire de la société et sa sociocritique du sujet transcendantal. Dans un deuxième temps, nous insisterons sur un aspect original de sa sociocritique, à savoir son infléchissement résolument politique.

\section{Les structures socio-aprioriques de la connaissance : «legein », " teukhein » et imaginaire social}

La sociocritique du sujet transcendantal se fonde chez Castoriadis sur une ontologie du social-historique. Il s'agit de réinscrire l'activité épistémique du sujet de la connaissance à l'intérieur des conditions imposées par le social-historique compris comme domaine sui generis de l'être. Cette enquête ontologique, à son tour, se déploie le long des deux axes principaux de son ontologie de l'être social-historique. Tout d'abord, il s'agit de rapporter l'activité du sujet à une condition transhistorique de toute institution social-historique, à savoir le «legein» et le «teukhein» - dimensions ensemblistes-identitaires de toute institution social-historique. Ensuite, Castoriadis entend rapporter le sujet à une seconde dimension de toute institution social-historique : l'imaginaire social comme tel. Conformément à la théorie de l'institution imaginaire de la société, comprendre le contenu et le sens de l'activité épistémique du sujet de la connaissance consiste donc, en partie, à les rapporter à ces deux structures socio-aprioriques de la pratique. Ce faisant, Castoriadis propose une variante holiste, centrée sur le concept d'imaginaire, du socioconstructivisme. 


\section{Le legein et le teukhein comme premières structures socio-aprioriques du sujet transcendantal}

En tant que création ontologique, en tant qu'émergence d'une nouvelle forme de l'être35, le social-historique se distingue de ce dont il émerge, notamment la nature. Mais l'irréductibilité du social-historique à la nature ne signifie pas qu'il ne l'ait pas pour condition nécessaire de possibilité. Au contraire, le social-historique ne peut pas exister en dehors d'une relation partielle à la nature, plus particulièrement à sa dimension ensembliste-identitaire, nommée par Castoriadis la " première strate naturelle ».

Il existe des contraintes « externes »- tout spécialement, celles imposées par la première strate naturelle. [...] Dans la mesure où la première strate naturelle contient, à un degré décisif, une dimension ensembliste-identitaire [...], l'institution sociale doit recréer cette dimension dans sa "représentation» du monde et d'elle-même, c'est-à-dire dans son monde propre, son Eigenwelt 36 .

Ainsi, l'avènement d'un monde social passe par la recréation de cette "première strate naturelle » dans et par la pratique sociale. Cette recréation, chaque fois particulière et propre à chaque société historique, est néanmoins un véritable invariant anthropologique. C'est à travers les concepts de legein et de teukhein que Castoriadis cherche à penser cette relation universelle de l'être social-historique à la dimension ensembliste-identitaire de la nature : le «teukhein est la dimension identitaire [...] du faire social ; le legein est la dimension identitaire du représenter/dire social37 [...]». C'est pourquoi les concepts de legein et de teukhein sont également définis comme «proto-institution[s] de toute société 38 ». En les désignant ainsi, Castoriadis veut dire que l'institution de la société comme telle se confond avec la création du legein et du teukhein comme dimensions ensemblistes-identitaires du dire et du faire. Ils sont « condition et création de la société, condition créée par ce qu'elle-même conditionne39 ». Aussi ne sont-ils pas la création d'individus singuliers, mais bien indissociables de l'auto-institution de la société elle-même : legein, teukhein et société sont cooriginaires, c'est-à-dire que la société n'existe pas en dehors de l'autocréation d'un système d'opérateurs et de catégories ensemblistes-identitaires.

Afin de spécifier la relation entre social-historique, legein, teukhein et première strate naturelle, Castoriadis parle également de relation d'《 étayage ». En empruntant librement ce concept à la métapsychologie freudienne, Castoriadis cherche à désigner

\footnotetext{
35 «La société est création, et création d'elle-même : auto-création. C'est l'émergence d'une nouvelle forme ontologique - d'un nouvel eidos - et d'un nouveau mode d'être. » Cornelius Castoriadis, «Imagination, imaginaire, réflexion », dans Les carrefours du labyrinthe, $V$, op. cit., p. 319.

36 Ibid., p. 321.

37 Cornelius Castoriadis, L'institution imaginaire de la société, op. cit., p. 521.

38 Cornelius Castoriadis, "Fait et à faire», dans Les carrefours du labyrinthe, V, op. cit., p. 64.

39 Cornelius Castoriadis, L'institution imaginaire de la société, op. cit., p. 330.
} 
une « relation sui generis » par laquelle une société recrée la dimension ensemblisteidentitaire de la nature. Avec le concept d'étayage, Castoriadis cherche à éviter deux écueils : le premier, qui consisterait à penser la création des catégories ensemblistesidentitaires par l'être humain selon une logique causaliste (la nature serait la cause des catégories, à la manière de l'empirisme ou du sensualisme le plus plat), ce qui conduit à occulter la pratique créatrice du sujet de la connaissance ; le second, qui consiste à la comprendre comme une objectivation herméneutique (le sujet de la connaissance révélerait des catégories présentes dans la nature), ce qui, là aussi, conduit à occulter la créativité du sujet de la connaissance. Contre l'empirisme et l'herméneutique, le concept d'étayage permet de dégager la spécificité de la relation du sujet de la connaissance à la nature : il crée un ensemble de déterminations épistémiques se trouvant correspondre à une strate de la nature ultimement inconnaissable en soi.

Dire que la logique identitaire peut être formulée et mise en œuvre équivaut donc à dire qu'il y a, qu'il existe effectivement des ensembles. Mais en même temps et aussi, il n'existe des ensembles que dans et par la logique identitaire, dans et par le legein. En ce sens, la logique identitaire, comme le legein, vaut décision ontologique sur ce qui est et la façon dont il est4o [...].

Castoriadis suit donc de très près la distinction centrale dans le criticisme kantien entre le phénomène et la chose en soi, à condition néanmoins de préciser, d'une part, qu'il la réarticule à une ontologie du social à travers laquelle une des origines de la catégorialité est le social-historique et, d'autre part, qu'il considère qu'on ne peut pas faire l'économie de l'hypothèse d'une organisation ensembliste-identitaire de la nature (sans quoi l'adéquation entre la pensée et la chose devient impensable). Ce dernier aspect est important, car il constitue un des pivots de la critique du kantisme par Castoriadis, au-delà de la perspective criticiste, voire même constructiviste, qu'il adopte par ailleurs. Selon lui, en effet, la thèse d'après laquelle il n'y a d'objectivité qu'à travers sa constitution transcendantale n'a aucune valeur si elle n'est pas accompagnée d'une réflexion plus profonde sur les caractères ontologiques de la « chose en soi ».

Enfin, il faut comprendre que cette institution social-historique du legein et du teukhein est elle-même la condition de possibilité de l'élaboration explicite de la rationalité ensembliste-identitaire par les logiciens et mathématiciens :

Les rudiments logiques de la théorie des ensembles [cantorienne, à partir de laquelle Castoriadis exhibe les schèmes distinctifs de la rationalité ensembliste-identitaire] importent ici parce que, quoi qu'il puisse en advenir du point de vue de la mathématique ellemême, ils condensent, explicitent et exemplifient de manière pure

40 Ibid., p. 335. 
ce qui, de tout temps, était sous-jacent à la logique identitaire et qui, longtemps avant que celle-ci n'ait reçu la moindre ébauche de formulation, constituait une dimension essentielle et inéliminable de toute activité et de toute vie sociale ${ }^{41}$.

En d'autres termes, les schèmes et opérateurs logiques fondamentaux qui se trouvent au fondement de la rationalité ensembliste-identitaire, brièvement commentés plus haut, sont avant tout des schèmes et opérateurs trouvant leur origine dans la pratique sociale, et c'est pourquoi le concept de rationalité ensembliste-identitaire est un concept praxéologique de la logique. L'autonomisation ultérieure de la rationalité ensembliste-identitaire dans l'histoire, point de départ de la constitution de la «pensée héritée », est donc à rapporter à la pratique des sujets individuels intéressés par la formalisation et l'abstraction du legein, bien qu'encouragée par l'institution nécessaire du social à travers la création d'une forme latente de rationalité ensembliste-identitaire :

Et l'emprise exercée sur nos esprits par les schèmes de cette pensée [héritée], produits d'un effort de trois mille ans de tant de génies incomparables, mais aussi [...] dans et par lesquels s'exprime, s'affine, s'élabore tout ce que l'humanité a pu penser depuis des centaines de milliers d'années et qui reflètent, en un sens, les tendances mêmes de l'institution de la société, ne pourrait être ébranlée, si tant est qu'elle puisse l'être, que par la démonstration précise et détaillée, cas après cas, des limites de cette pensée et des nécessités internes, d'après son mode d'être, qui l'ont amenée à occulter ce qui me paraît essentiel [à savoir la création $\left.{ }^{2}\right]$.

Le legein et le teukhein sont donc des structures socio-aprioriques de l'agir humain, chaque fois recréées de manière inédite par chaque société dans l'histoire. Si nous voulions enfermer la thèse de Castoriadis dans un ensemble de dénominations, nous pourrions dire qu'il propose, en ce qui concerne l'origine des catégories logiques, une variante holiste (la création du sujet est à rapporter à ses conditions socialhistoriques), pragmatique (les catégories logiques ont une origine pratique) et universaliste (chaque système logique est historiquement singulier, mais toute société fait face à la nécessité d'en créer un) du socioconstructivisme : la création historique des catégories logiques, celles qu'il regroupe d'après la dénomination d' " ensemblisteidentitaire », a pour condition fondamentale de possibilité l'être social-historique luimême, lequel se déploie universellement le long de ces deux dimensions que sont le «legein» et le «teukhein». À leur tour, celles-ci sont à comprendre comme recréation d'un ensemble catégoriel, conditionnée mais non déterminée, par une immanence naturelle de la catégorialité logique. Aussi l'historicité de la connaissance, qui est

${ }^{41}$ Ibid., p. 329.

42 Ibid., p. 6-7. 
en partie celle de ses catégories logiques, se loge dans ce triangle relationnel dont chacun des sommets est susceptible de se transformer: autoposition du socialhistorique; créativité propre du sujet de la connaissance; immanence naturelle de l'ensembliste-identitaire.

\section{L'imaginaire social comme seconde structure socio-apriorique du sujet transcendantal}

La seconde structure socio-apriorique de l'agir et de la pensée est l'imaginaire social - inextricablement mêlée à sa dimension ensembliste-identitaire, bien que lui étant distincte. Celui-ci, indescriptible à partir des schèmes et catégories de la pensée héritée, est ce qui singularise véritablement le social-historique d'un point de vue ontologique : le social-historique, c'est l'imaginaire social, et chaque société singulière est à rapporter à la spécificité des créations de son imaginaire social propre. « Nous devons reconnaître que le champ social-historique est irréductible aux types traditionnels d'être, que nous observons ici les œuvres, la création de ce que j'appelle l'imaginaire social ou la société instituante 43 [...]. » C'est pourquoi la tâche de la pensée sociologique et historique doit être de développer la rationalité adéquate à l'expression, en dehors des schèmes de la rationalité ensembliste-identitaire, du mode d'être de l'imaginaire social et des relations qu'il entretient avec les autres niveaux ontologiques dont il est voisin, notamment le psychique et la nature44. Quoi qu'il en soit, un des rôles principaux que Castoriadis assigne à l'imaginaire est la création d' « images », " position-création de figures comme présentification de sens et de sens comme toujours figuré-représenté $45 »$. Cela signifie que l'imaginaire social, distinct de l'imagination radicale comme versant proprement individuel de l'imaginaire, crée de manière immotivée des « images », des « figures », servant de médiations aprioriques à la constitution de l'objectivité et de sa signification. Ces principes socio-aprioriques de médiation de l'expérience, principes à partir desquels l'expérience est constituée pour l'individu socialisé et pour la société en tant qu'être pour-soi, sont ce que Castoriadis appelle les « significations imaginaires sociales». L'imaginaire social, c'est essentiellement cela : la source impersonnelle, distinctive du champ social-historique, de la création d' « images », de « figures », qui font office de médiations socio-aprioriques de l'expérience. Comme l'écrit Castoriadis, les « significations imaginaires sociales » sont ce par quoi les «"objets" et donc aussi la

43 Cornelius Castoriadis, "L'imaginaire : la création dans le domaine social-historique », dans Les carrefours du labyrinthe, II, op. cit., p. 280.

44 « De même que lorsque nous parlons du social-historique et de l'imaginaire social, la difficulté n'est pas d'inventer de nouveaux vocables pour ce qui est ici en cause, mais de comprendre que ce que ces vocables visent n'est pas catégorisable moyennant les catégories grammaticales (et, derrière elles, logiques et ontologiques) selon lesquelles nous sommes habitués à penser. » Cornelius Castoriadis, L'institution imaginaire de la société, op. cit., p. 532.

45 Ibid., p. 533. 
relation de "référence" sont rendus possibles 46 ». Plus largement, elles sont les principes socio-aprioriques à partir desquels s'institue le monde propre d'une société considérée :

La rupture radicale, l'altération que représente l'émergence du social-historique dans la nature pré-sociale est la position de la signification et d'un monde de significations. La société fait être un monde de significations et est elle-même par référence à un tel monde 47 .

En ce sens, elles ne sont pas uniquement des principes épistémiques, mais elles sont aussi une des origines de la signification affective que l'individu se fait du monde. Les « significations imaginaires sociales » assument donc un rôle bien plus large que la simple constitution d'une objectivité scientifique. Elles sont les «images », les « figures » à partir desquelles le monde est expérimenté dans sa totalité par la société comme être pour-soi et pour les individus historiquement situés, impliquant par ailleurs un certain rapport affectif et émotionnel à celui-ci.

En outre, elles ne sont pas seulement des médiations socio-aprioriques de l'expérience par lesquelles est qualifié qualitativement le réel, mais elles sont aussi les principes de totalisation du social-historique lui-même. Cela signifie, si nous voulons reprendre le vocabulaire kantien, que les significations imaginaires sociales assument, pour chaque société considérée, une fonction de synthèse transcendantale.

Ce qui tient une société ensemble, c'est le tenir ensemble de son monde de significations. Ce qui permet de la penser dans son eccéité, comme cette société-ci et pas une autre, c'est la particularité ou la spécificité de son monde de significations imaginaires sociales, organisé ainsi et non autrement 48 .

L'origine kantienne de la définition castoriadienne des significations imaginaires sociales a trop peu souvent été remarquée, bien qu'elle soit aussi déterminante que son ancrage merleau-pontien ou lacanien 49 (dont les définitions de l'imaginaire comme structure apriorique de l'expérience sont d'ailleurs impossibles sans la réorientation criticiste de la philosophie par Kant). Dans la Critique de la raison pure, Kant définit l'imagination (Einbildung) comme la faculté transcendantale permettant, d'une part, la synthèse unitaire du divers de l'intuition et, d'autre part, la subsomption du divers de l'intuition aux concepts purs de l'entendement par l'intermédiaire

\footnotetext{
46 Ibid., p. 527.

47 Ibid., p. 519.

48 Idem.

49 Par exemple dans les interprétations proposées par N. Poirier et W. Breckman. $C f$. Nicolas Poirier, L'ontologie politique de Castoriadis : création et institution, Paris, Payot, 2011 ; Warren Breckman, Adventures of the symbolic: post-Marxism and radical democracy, New York, Columbia University Press, 2013.
} 
de ses schèmes transcendantaux ${ }^{\circ}$. Pour Castoriadis, le schématisme transcendantal est une des plus grandes découvertes de Kant, dans le sens où il permet de penser le rôle de l'imagination dans la constitution de l'objectivité : nulle expérience possible en dehors des schèmes, qui sont des images, fournis par l'imagination. " Le point le plus fort - et le plus vrai - de la conception kantienne de l'imagination est évidemment l'idée du schématisme comme médiation entre les catégories et les "données sensorielles51" ». Tout comme Kant, Castoriadis pense que l'objectivité ne peut se constituer qu'à travers cette médiation apriorique qu'est l'« image » produite par l'imagination - qui n'est d'ailleurs pas une " image » au simple sens usuel du terme, mais dans le sens d'une forme où viennent se couler l'expérience et la perception. Ainsi la distinction que fait Castoriadis entre une imagination seconde et une imagination première recoupe-t-elle d'assez près celle que fait Kant entre l'imagination productive (transcendantale) et reproductive (celle dont traite la psychologie ${ }^{2}$ ).

Ceci remarqué, il existe de nombreuses différences entre les conceptions castoriadienne et kantienne de l'imagination transcendantale53. La plus évidente est la pluralisation ontologique des manifestations de l'imagination chez Castoriadis, puisqu'il n'en existe pas chez Kant d'espèce spécifiquement sociale et historique. Cette diversification des genres de l'imagination ouvre un problème absent du criticisme kantien, celui de l'articulation entre les différentes modalités de la constitution transcendantale de l'expérience. Comment doit-on penser l'articulation entre la constitution socioapriorique de l'objectivité par l'intermédiaire des significations imaginaires sociales, d'un côté, et celle découlant des schèmes fournis par l'imagination radicale (individuelle), de l'autre côté ? La deuxième différence importante est, comme le remarque

$5^{0}$ Emmanuel Kant, Critique de la raison pure, trad. Alain Renaut, Paris, Flammarion, 2006, p. 224-227.

${ }^{1}$ Cornelius Castoriadis, «Imagination, imaginaire, réflexion », dans Les carrefours $d u$ labyrinthe, $V$, op. cit., p. 287.

52 Ibid., p. 271.

53 On trouvera dans «Imagination, imaginaire, réflexion» la discussion la plus rigoureuse de Castoriadis avec la conception kantienne de l'imagination. Nous ne le ferons pas ici, mais il y aurait une étude comparative très éclairante à mener à ce sujet entre Castoriadis et Heidegger. Il est en effet frappant que la réintroduction de la question ontologique au-delà du criticisme kantien s'articule, aussi bien chez Castoriadis que chez Heidegger, à un commentaire critique du rôle alloué par Kant à l'imagination transcendantale dans la constitution de l'expérience. Castoriadis était d'ailleurs un lecteur attentif de cette partie de l'œuvre heideggérienne. Il s'y réfère par exemple en notes (Ibid., p. 288, n. 17), ou bien la critique (Cornelius Castoriadis, «La découverte de l'imagination », dans Les carrefours du labyrinthe, II, op. cit., p. 413). On notera par ailleurs que S. Zizek a déjà écrit quelques pages non sans intérêt sur les rapports entre Castoriadis, Heidegger et l'imagination transcendantale kantienne. Il clôt son (bref) commentaire comparatif sur une affirmation péremptoire jurant avec la finesse de ses analyses précédentes, et qui ne signifie d'ailleurs pas grand-chose à défaut d'être mieux développée : « La notion d'imagination de Castoriadis reste cependant prise dans l'horizon existentialiste de l'homme en tant qu'être qui projette son "essence" dans l'acte d'imagination qui transcende tout Être positif. »Cf. Slavoj Zizek, Le sujet qui fâche : le centre absent de l'ontologie politique, Paris, Flammarion, 2007, p. 35-36. 
opportunément Castoriadis en s'appuyant en partie sur Fichte54, que l'imagination transcendantale kantienne n'est pas créatrice: elle ne crée pas les schèmes qu'elle pourvoie à l'intuition et à l'entendement, et elle n'est dotée, par conséquent, d'aucune initiative historique : «Si l'imagination transcendantale commençait à imaginer quoi que ce soit, le monde de Kant s'effondrerait aussitôt. Pour cette raison encore, Kant ne peut ou ne veut pas voir la fonction créatrice de l'imagination dans le domaine cognitif (scientifique ou philosophique55). » Il s'agit, au contraire, de prendre acte du fait que l'imagination (dans son versant social comme imaginaire et dans son versant individuel comme imagination radicale) a une histoire, celle de la création immotivée de nouveaux schèmes socio-aprioriques à partir desquels le réel est constitué. On voit dès lors l'abîme épistémologique qu'ouvre Castoriadis en réarticulant la notion d'imagination (collective et/ou individuelle) à celle de création : il s'agit à la fois de penser les différents niveaux d'articulation de la constitution de l'objectivité (indiscernablement individuelle et collective) en dehors du référentiel ensemblisteidentitaire et la variation historique de cette constitution d'après la modalité de la création ex nihilo - cette création étant celle de nouvelles " figures du pensable ». Enfin, cet abîme devient proprement vertigineux lorsqu'on rapporte la réflexion épistémologique de Castoriadis à sa réflexion ontologique : comment penser, en effet, l'articulation entre l'historicité de ce sujet de la connaissance, lequel se constitue au carrefour de l'imagination radicale et de l'imaginaire social, et celle de l'être compris comme émergence toujours imprévisible de nouvelles formes dans le temps ?

La spécificité de la sociocritique du sujet transcendantal chez Castoriadis tient donc à la manière dont il la réarticule à une ontologie du social-historique centrée sur le concept d'imaginaire. Il ne s'agit pas seulement de dire qu'il existe une origine sociale de la connaissance, mais que celle-ci doit être systématiquement rapportée à «l'imaginaire social », principe apriorique de synthèse des interactions sociales et de constitution de la phénoménalité. Là encore, Castoriadis propose une variante holiste et pragmatique du socioconstructivisme : la pratique épistémique, qui est une construction sociale, doit être rapportée aux conditions imposées par l'être socialhistorique, lesquelles s'énoncent dans la position apriorique de la signification imaginaire sociale. Ainsi, l'histoire de la science est, en partie (nous y reviendrons en conclusion), celle des mutations des imaginaires dans lesquels se déploient les pratiques épistémiques.

\section{Une variante politique de la critique sociologique du sujet transcendantal}

Si la question de la sociogenèse du sujet de la connaissance (celle consistant à identifier les structures sociales desquelles découlent les catégories mobilisées lors de

54 Cornelius Castoriadis, «L'état du sujet aujourd'hui », dans Les carrefours du labyrinthe, III, op. cit., p. 247-248.

55 Cornelius Castoriadis, «Imagination, imaginaire, réflexion », dans Les carrefours du labyrinthe, $V$, op. cit., p. 286. 
l'activité épistémique) est centrale pour toute sociocritique du sujet transcendantal, celle de l'individuation des catégories sociales l'est tout autant: par quelles médiations l'individu parvient-il à s'approprier les catégories mises à disposition par le social et à en faire usage lors de ses pratiques épistémiques ? On ne trouve pas chez Castoriadis, contrairement, par exemple, à l'épistémologie génétique piagétienne ou au constructivisme vygotskien, une réflexion approfondie sur cette question. Nous pouvons lire chez Castoriadis une réflexion élaborée sur la socialisation de la psyché56, mais nous ne trouvons pas de réflexion précise sur l'appropriation par l'individu des catégories de la connaissance, qui est un aspect plus circonstancié de la question de la socialisation de la psyché.

Cette absence se comprend aisément si on se rapporte à l'intention du projet épistémologique de Castoriadis. En effet, il s'agissait moins pour lui d'élaborer une théorie de l'individuation et des médiations par lesquelles s'opère la sociogenèse de la catégorialité que d'" élucider» les conditions où elle se déploie afin d'ouvrir la science à sa propre pratique créatrice. Or, si on se concentre sur cette deuxième question, nous pouvons voir que Castoriadis propose davantage qu'une simple conception sociogénétique de la connaissance, puisqu'il rapporte la pratique scientifique à certaines de ses conditions politiques. Ainsi, l'originalité de la sociocritique du sujet transcendantal chez Castoriadis ne tient-elle pas seulement à son articulation à la théorie de l'institution imaginaire de la société, mais aussi à son inscription dans une conception de l'histoire centrée autour du « projet d'autonomie ». Ce qui est en jeu, c'est l'avènement d'un sujet réflexif du savoir, c'est-à-dire d'un sujet créateur de ses propres déterminations transcendantales - et non seulement un sujet automate reconduisant les déterminations imposées par l'être social-historique.

En effet, outre son ancrage sur une théorie sociale originale, la théorie de l'institution imaginaire de la société, la seconde grande spécificité du socioconstructivisme castoriadien est de rapporter la constitution du sujet, c'est-à-dire la forme spécifique de pour-soi capable de retourner sur ses propres déterminations transcendantales et de les créer consciemment, à des conditions politiques uniques : la retotalisation du champ social d'après le projet d'autonomie politique sous la forme d'une constitution démocratique. Pour Castoriadis, il n'y a de sujet à proprement parler qu'à condition que le champ social se soit ouvert à la critique de ses fondements normatifs. En ce sens, il est possible de dire que l'avènement du sujet de la science comme créateur de ses propres déterminations transcendantales est une forme de subjectivation démocratique. Ce sont donc quelques aspects de cette thèse que nous

$5^{6}$ On pourra lire : Cornelius Castoriadis, L'institution imaginaire de la société, op. cit., chap. VI ; Cornelius Castoriadis, "Psyché et éducation », dans Les carrefours du labyrinthe, VI. Figures du pensable, Paris, Seuil, 2009, p. 239-268 ; Cornelius Castoriadis, "À nouveau sur la psyché et la société », dans Les carrefours du labyrinthe, VI, op. cit., p. 289-314; Cornelius Castoriadis, "L'état du sujet aujourd'hui », dans Les carrefours du labyrinthe, III, op. cit. ; Cornelius Castoriadis, La création humaine, I. Sujet et vérité dans le monde social-historique. Séminaires 1986-1987, Paris, Seuil, 2002. 
allons commenter désormais, en prenant notamment pour fil rouge la question de l'émergence d'un « projet de vérité », dont le sujet est le porteur rationnel.

La " clôture " comme position transcendantale initiale

La modalité initiale d'après laquelle la réalité est co-constituée par le socialhistorique et le psychique est celle de la "clôture ", dans le sens où le champ socialhistorique et l'individu socialisé ne sont pas de facto prédisposés à la reprise critique des concepts, catégories et a priori par lesquels l'objectivité est construite.

[...] ce qui caractérise l'immense majorité des sociétés est que ces questions [celles relatives à la constitution de l'objectivité] restent toujours limitées, ne peuvent pas dépasser, ni même atteindre, viser, ce que sont pour la société, pour la tribu, ce qu'on appellerait métaphoriquement les axiomes de l'institution sociale, ses règles d'inférence et ses critères déductifs 57 .

De ce point de vue, les individus socialisés et le champ social-historique sont des modalités comme une autre de la constitution du réel comme «pour-soi». Castoriadis soutient dans une perspective constructiviste que la phénoménalité se constitue toujours pour un être vivant singulier, qu'il s'agisse de la cellule, de l'individu, ou d'une société, et ce, d'après les limites et conditions imposées par les lois de son auto-organisation formelle. Mais la constitution du réel comme "poursoi » ne signifie pas pour autant que les médiations par lesquelles le réel est constitué soient présentes à la conscience ou à la réflexion du "pour-soi» considéré58. En d'autres termes, l'autoréférentialité, c'est-à-dire la position transcendantale sans laquelle le réel ne peut pas être constitué, n'est pas encore la réflexivité comme conscience, et éventuellement transformation, des déterminations transcendantales de l'objectivité.

De la « clôture » à la réflexivité transcendantale : la condition politique

La thèse de Castoriadis est que le passage de l'autoréférentialité à la réflexivité a pour condition nécessaire la retotalisation du champ social d'après la signification de l'autonomie politique. Comme l'écrit Castoriadis,

La philosophie [comprise ici comme forme inaugurale du «projet de vérité »] naît dans la polis et ne peut naître que dans la polis.

\footnotetext{
57 Cornelius Castoriadis, "Imagination, imaginaire, réflexion », dans Les carrefours du labyrinthe, $V$, op. cit., p. 327.

58 « Si l'on considère le système immunitaire, le reckoning, le calcul, la computation y sont évidemment constamment présents ; mais tout autant, l'autoréférence. Le système immunitaire n'est rien s'il n'est capacité permanente [...] de distinguer le soi du non-soi [...]. Dans la réflexivité nous avons quelque chose de différent : la possibilité que la propre activité du "sujet" devienne "objet" [...]. » Cornelius Castoriadis, «L'état du sujet aujourd'hui », dans Les carrefours du labyrinthe, III, op. cit., p. 261-262.
} 
Polis doit être pris ici au sens essentiel : une collectivité humaine qui tend à s'autogouverner et à s'auto-instituer. La philosophie elle-même n'est qu'une dimension de cet effort visant à l'autoinstitution, puisqu'elle est refus des représentations simplement héritées, simplement instituées, et prétention d'établir les représentations vraies par l'activité autonome de la pensée humaine59.

C'est en suivant les développements qu'il propose sur l'essor d'un "projet de vérité » dans Sujet et vérité dans le monde social-historique que nous pouvons nous en convaincre.

Dans Sujet et vérité dans le monde social-historique, Castoriadis commence par "trivialiser» la définition de la vérité comme convenance6o. Le vrai comme « convenance » est la reformulation par Castoriadis de la théorie classique de la vérité comme adéquation. Selon celle-ci, une proposition émise par un individu est «vraie » lorsque son contenu est adéquat aux propriétés intrinsèques d'un état de choses visé par la proposition. Castoriadis préfère parler de «convenance » plutôt que d'adéquation, notamment afin de marquer la relativité des principes ultimes (les significations imaginaires sociales) qui orientent les propositions linguistiques ayant une prétention à la vérité : est "vraie » une proposition lorsque son contenu convient, d'une part, aux propriétés de l'objet qu'elle vise, et, d'autre part, lorsqu'elle convient aux critères socialement admis du vrai. Une fois cette conception du vrai admise, qui serait universelle, Castoriadis soutient que l'émergence de la raison, associée à l'émergence de la philosophie dans une société démocratique, est conservation et dépassement du modèle du vrai comme convenance ${ }^{61}$. Elle est « conservation », car il ne saurait y avoir de raison en dehors du rapport du sujet à son objet caractéristique de la vérité comme convenance ; mais elle est ipso facto « dépassement », car elle est ouverture à une interrogation illimitée portant sur les critères admis du vrai. La raison portée par le sujet de la science est dès lors définie comme un mouvement, comme une dynamique visant l'évaluation et la création des critères de la vérité qui vaudront ensuite dans le cadre du rapport du sujet à l'objet comme convenance. La raison est « création de résultats, création de méthodes et création de critères - sans que, finalement, l'on puisse prétendre s'arrêter et dire : voilà le critères [sic] des critères, ce n'est que d'après ce qui est posé par ce métacritère ou cette métanorme que l'on peut

59 Cornelius Castoriadis, La création humaine, I. Sujet et vérité dans le monde social-historique. Séminaires 1986-1987, op. cit., p. 316.

60 On peut lire: Philippe Caumières, «La vérité comme création social-historique », dans Cahiers Castoriadis, VI. Castoriadis et la question de la vérité, dir. Philippe Caumières, Sophie Klimis, Laurent Van Eynde, Bruxelles, Facultés Universitaires Saint-Louis, 2006, p. 9-33 ; Arnaud Tomès, " Politique et vérité chez Castoriadis », dans Cahiers Castoriadis, VI. Castoriadis et la question de la vérité, op. cit., p. 53-66.

${ }^{61}$ Cornelius Castoriadis, La création humaine, I. Sujet et vérité dans le monde social-historique. Séminaires 1986-1987, op. cit., p. 304. 
décider du vrai62 ». La raison, autrement dit, est une métarationalité visant la redéfinition des critères du « vrai » par création et évaluation des normes du «vrai » qui s'appliqueront ensuite.

Que peut-on dire, dès lors, sur la vérité comme caractéristique d'une pensée ? C'est précisément une caractéristique non pas d'un énoncé ou d'une pensée, mais d'un mouvement de pensée. C'est le mouvement incessant, ou l'effort incessant pour mettre en cause ce que nous avons appelé provisoirement à notre tour la phénoménalité de l'objet ${ }^{63}$.

Ailleurs dans son séminaire, Castoriadis explicite le rapport que sa définition de la raison entretient avec la question de la subjectivité transcendantale. Il précise que « le mouvement de la vérité » est aussi redétermination des limites de la subjectivité, c'est-à-dire explicitation par le sujet connaissant de ce qui lui appartient comme sujet connaissant, et constitution phénoménale de l'objet. La raison est clairement conçue comme une activité subjectivante dont la fonction est de tracer la ligne de partage entre le sujet et l'objet, mais aussi de retracer cette ligne selon l'expérience que fait le sujet de son objet.

Le mouvement de la vérité, dans l'histoire de la pensée, est la tentative d'éprouver le plus possible les limites [...], de reculer les murs, de distendre la clôture pour voir ce qui, dans ce que l'on pense, vient de l'activité de pensée elle-même et ce qui vient de son extérieur. Le mouvement de la vérité est l'effort de dépasser la phénoménalité, et la vérité est l'ébranlement de la phénoménalité qui en est la résonnance 64.

L'activité de la raison comme reprise critique des principes de la connaissance est comprise ici comme une véritable expérience à travers laquelle le sujet de la connaissance se définit lui-même dans son rapport à l'objet. En explicitant les principes qui déterminent son rapport à l'objectivité, il délimite les contours et la forme de sa propre subjectivité, mais aussi celles de la phénoménalité. Ainsi, la connaissance est une pratique où le sujet se fait comme sujet connaissant. L'histoire de la connaissance est celle de la création (non dialectique) des formes de la subjectivité transcendantale.

C'est donc cette différence entre une pratique de la vérité comme adéquation et une pratique autonome de la vérité qui fonde celle entre le simple individu socialisé et le sujet : le sujet, au sens strict du terme selon Castoriadis, est l'individu doté de la capacité réflexive lui permettant d'inspecter les fondements aprioriques par lesquels il expérimente le réel, ce qui vaut aussi bien pour la science que d'autres domaines de l'activité.

62 Ibid., p. 306.

63 Ibid., p. 314.

64 Ibid., p. 315-316. 
[E]n règle générale [...], il [l'être humain] n'est pas en mesure de mettre ces cadres [les cadres imposés par le social-historique] en question ni par conséquent de se mettre en question lui-même. Il n'a pas donc la réflexivité au sens strict et fort du terme [...], caractéristique de ce qu'il faut appeler la subjectivité humaine. De celle-ci on doit dire que, création historique relativement récente [...], elle est une virtualité de tout être humain, certainement pas une fatalité. [...] C'est en tant qu'il se fait subjectivité que l'être humain peut se mettre en cause et se considérer comme origine, certes partielle, de son histoire passée, comme aussi vouloir une histoire à venir et vouloir en être le co-auteur65.

Nous voyons donc que selon Castoriadis la possibilité pour le sujet de transformer réflexivement sa propre catégorialité transcendantale n'est pas donnée universellement. Il s'agit, au contraire, d'une situation épistémique singulière et socialhistoriquement déterminée. Ce n'est que dans une société valorisant collectivement l'inspection critique des fondements de la pratique et de la pensée qu'un tel sujet de la connaissance peut advenir. Aussi le sujet en tant qu'individu autonome est-il la forme spécifique de l'individualité correspondant à une société elle-même autonome. Cette société, c'est la société démocratique.

\section{Conclusion}

La sociocritique du sujet transcendantal par Castoriadis s'inscrit dans une tradition bien établie et qu'on peut faire remonter à la naissance des sciences sociales et historiques. Nous avons vu notamment qu'il reproduit la structure argumentative distinctive de la variante " holiste " de la sociocritique du sujet transcendantal. Il s'agit de penser le champ social comme une condition transcendantale du sujet transcendantal, plus particulièrement de proposer un cadre conceptuel expliquant l'origine sociale de la catégorialité mobilisée par le sujet de la science lors de ses activités épistémiques. Aussi Castoriadis pense-t-il qu'on ne peut expliquer les rapports entre individus et société qu'à condition de penser le champ social comme une "réalité sui generis ", c'est-à-dire de laisser une place au champ social et historique à l'intérieur d'une ontologie pluraliste - réactivant ainsi la classification d'origine aristotélicienne des genres de l'être et faisant de la sociologie la science étudiant le genre « social» - ce qui requiert, d'après lui, une critique des savoirs déjà constitués sur le social et l'histoire. C'est la raison pour laquelle nous avons examiné l'écart considérable séparant la conception castoriadienne des sciences sociales et la tradition sociologique. Castoriadis, en effet, réinscrit sa théorie de l'institution imaginaire de la société, qui est sa théorie sociale à partir de laquelle il pense la constitution du sujet transcendan-

65 Cornelius Castoriadis, «L'état du sujet aujourd'hui », dans Les carrefours du labyrinthe, III, op. cit., p. 276-277. 
tal, à l'intérieur d'un projet philosophique plus ambitieux, à savoir la critique des conséquences de l'universalisation d'une forme restreinte de rationalité, celle dite « ensembliste-identitaire ». L'ancrage de sa théorie sociale dans ce projet philosophique le conduit, comme nous l'avons vu, à poser un regard critique sur l'histoire des sciences sociales et historiques, y compris sur les variantes « holistes » de ces disciplines. La critique des sciences sociales par Castoriadis se présente alors comme une critique de la "pensée héritée » dans les sciences sociales et consiste à montrer comment leur fondation sur les schèmes et opérateurs logiques distinctifs de la rationalité ensembliste-identitaire menace leur légitimité et leur pertinence scientifique. Proposant en contrepartie une théorie du social centrée sur la notion d'imaginaire, Castoriadis reprend la question de la constitution du sujet transcendantal de façon originale dans un dialogue étroit, parfois implicite, avec la conception kantienne du schématisme exposée dans la Critique de la raison pure. Une autre originalité de cette critique, nous l'avons également examiné, est sa mise en perspective avec la question de l'autonomie politique.

Bien évidemment, ce qui est en jeu dans cette sociocritique du sujet transcendantal est la question de la vérité. L'exposé que nous avons fait de cet aspect de l'œuvre de Castoriadis, dans lequel nous avons accentué l'ancrage de sa philosophie dans celle de Kant, pourrait laisser penser que Castoriadis propose une variante comme une autre de socioconstructivisme, voire de relativisme, où la vérité ne serait que la propriété d'un énoncé linguistique déterminée par ses conditions social-historiques de possibilité. Or, Castoriadis était particulièrement critique à l'égard de ces conceptions de la science qui, à l'instar de celles de Kuhn ou de Foucault (du moins est-ce l'interprétation qu'il en fait), évacuent la question de la «chose en soi » afin de se concentrer sur la seule question transcendantale. Contre Foucault, notamment, Castoriadis faisait valoir que la problématisation des conditions sociales du savoir problème que Foucault cherchait à résoudre dans Les mots et les choses à travers le concept d'épistémè - ne peut pas évacuer celle, ontologique, des propriétés fondamentales de l'être/étant désigné par le savoir, aussi socialement déterminé soit-il.

Poser ces questions [celles portant sur les conditions sociales du savoir], c'est donc, bien sûr, s'interroger sur le contenu du " savoir scientifique " à chaque étape ou à chaque époque ; mais c'est aussi, évidemment, s'interroger sur ce qui est ainsi chaque fois connu, autrement dit sur l'organisation et le contenu de ce qui, simplement, est. Si cette question n'est pas soulevée, si les «paradigmes » (ou les épistémè) successifs sont posés de façon purement descriptive, sans aucune interrogation concernant leur relation réciproque et ce qui, dans l'objet qu'ils visent, rend possible leur existence, leur succession et leur succession dans cet 
ordre et non pas dans n'importe quel autre, on ne réfléchit pas vraiment la science, on n'en fait au mieux que l'ethnographie ${ }^{66}$.

L'intention de Castoriadis n'était donc pas de proposer une critique de la raison où elle serait destituée de sa capacité à énoncer une vérité universelle. Il s'agissait plutôt d'ouvrir la science à sa propre pratique en explicitant les conditions ontologiques dans lesquelles elle se déploie. La thèse d'une constitution sociale de la réalité chez Castoriadis n'est pas évacuation d'une conception universaliste de la vérité, mais élucidation d'une de ses conditions de possibilité, à savoir celle ayant trait à la dimension subjective de la relation entre subjectivité et objectivité, laquelle doit par ailleurs être réinscrite à l'intérieur d'une conception cosmologique plus large valorisant la contingence et la pluralité de la création. La sociocritique du sujet transcendantal par Castoriadis ne vise donc pas la relativisation et l'égalisation des formes de discursivité, mais un gain de réflexivité scientifique, notamment en l'exposant à certaines propriétés fondamentales de l'être qui auraient été occultées à ce jour : la création comme émergence immotivée de nouvelles formes de l'être dans le temps et la pluralité qualitative de ses registres organisationnels.

66 Cornelius Castoriadis, "Science moderne et interrogation philosophique ", dans Les carrefours $d u$ labyrinthe, I, op. cit., p. 219-220. 